\title{
Can We Trust the Conclusion "No Significant Difference" in Plastic Surgery Trials?
}

\author{
Osama A. Samargandi ${ }^{1}$ (D) Alex Koziarz $^{2} \cdot$ Ahmad Makhdoum $^{3} \cdot$ Lujain Mirdad $^{4}$

Received: 19 November 2020/Accepted: 13 December 2020/Published online: 6 January 2021

(C) Springer Science+Business Media, LLC, part of Springer Nature and International Society of Aesthetic Plastic Surgery 2021

Level of Evidence $V$ This journal requires that authors assign a level of evidence to each article. For a full description of these Evidence-Based Medicine ratings, please refer to the Table of Contents or the online Instructions to Authors www.springer.com/00266.

Before embarking on a clinical study that aims to evaluate the efficacy of an intervention, a researcher must determine the adequate sample size needed to produce a reliable conclusion. The probability of obtaining a positive result for a dichotomous outcome at a pre-specified alpha threshold depends on the control arm's event rate, minimal clinically significant difference, and enrolment ratio. Lower sample sizes increase the probability of negative findings at risk of type 2 error [1,2]. In other words, with a larger sample size, there may in fact be a statistically significant difference that clinicians would consider for future patient care. Failure to identify this difference translates to some interventions not offered to patients due to previous methodological limitations.

Osama A. Samargandi

Osamargandi@dal.ca

1 Division of Plastic Surgery, Faculty of Medicine, Dalhousie University, Room 4447, Halifax Infirmary, 4th Floor, Plastic Surgery, 1796 Summer Street, Halifax, NS B3H 3A7, Canada

2 Faculty of Medicine, University of Toronto, Toronto, ON, Canada

3 Division of Cardiac Surgery, University of Toronto, Toronto, ON, Canada

4 Faculty of Dentistry, Dalhousie University, Halifax, NS, Canada
The evidence in plastic surgery is trending towards conducting and publishing more randomized controlled trials (RCTs) [3]. RCTs offer the advantage that allowing for balance of known and unknown prognostic factors may otherwise influence outcomes between two treatments. Unfortunately, published RCTs in the plastic surgery field have lacked proper sample calculations, particularly when designing the trial [4-7]. Voineskos et al. [4, 5] conducted a systematic review of 173 RCTs in plastic surgery and found that $75 \%$ of trials did not report an a priori sample size calculation. Similarly, Chung et al. [7] analyzed the power of 111 controlled studies that reported negative findings and suggested that a high number of these studies are underpowered. In addition, Ayeni et al. [8] reported in their review of plastic surgery literature that only $19 \%$ of trials performed an a priori power analysis. They concluded that reporting of power analysis has slightly increased over the last decade; however there is still room for improvement [8].

The lack of power analysis may be correlated with the suboptimal reporting in plastic surgery research [9]. The use of reporting guidelines or checklists can provide guidance on the important standardized information needed in a research paper. For example, the Consolidated Standards of Reporting Trials (CONSORT) criteria are the most popular checklist used for RCTs [10]. The criterion number "7a" assesses how sample size should be determined in the a study. The Strengthening the Reporting of Observational Studies in Epidemiology (STROBE) checklist performs a similar function but for observational studies, recommending authors whether and how sample size was calculated [11]. Therefore, reporting guidelines are recommended for clinical trialists when designing RCTs, 
Table 1 Summary of recommendations to improve the quality of reporting of trials in plastic surgery

\begin{tabular}{ll}
\hline Recommendations & \\
\hline For Authors & $\begin{array}{l}\text { Consult an epidemiologist and a biostatistician early when drafting a study protocol and analysis } \\
\text { Specify a priori primary and secondary outcomes, preferably in a published protocol }\end{array}$ \\
& Use an appropriate reporting checklists (e.g. CONSORT [10], STROBE [11]) \\
For Journal Editors & $\begin{array}{l}\text { Instruction for authors sheet should guide authors that the statement "no significant difference results" in RCTs should be } \\
\text { accompanied by an a priori power analysis } \\
\text { Instruct authors to use and submit reporting checklists (e.g. CONSORT [10], STROBE [11]) }\end{array}$ \\
$\begin{array}{l}\text { For Journal } \\
\text { Reviewers }\end{array}$ & $\begin{array}{l}\text { Instruct authors to report the sample size calculation, or mention that this step was not performed in the limitation section } \\
\text { with a justification of not doing so }\end{array}$ \\
\hline
\end{tabular}

and may serve as an important tool in the design of future plastic surgery trials.

RCTs involve several methodological steps to overcome biases that may otherwise lead to inaccurate results. Biases such as confounding are generally not considered to explain lack of significance difference in RCTs assuming random sequence generation and allocation concealment were properly conducted [3]. Thus, when the results of an RCT are negative, the first suspicion that may arise in an investigator's mind is whether or not the sample size was sufficient. Unlike RCTs, observational studies lack randomization. In plastic surgery, many observations are also retrospective, which rarely included blinded outcome assessments. Therefore, they are at a higher risk of multiple biases and their results could potentially be confounded by many factors. The effect of confounders on the results is commonly addressed during the analysis phase using multivariable regression analyses. Large sample sizes are strongly recommended when investigators plan to evaluate several covariates, require multi-level modeling, or have a low event rate for the outcome of interest.

To conclude, there is generally more trust in lack of significant difference between treatments in a clinical trial if a reasonable a priori power analysis was performed. Future clinical trials in plastic surgery are urged to perform a priori power analyses using robust and transparent methodology that allows for replication. This dedication to a meticulous methodology during early phases of trial design reduces the risk of future, redundant studies which waste healthcare resources and harm patients, which can harm patients and be a waste of healthcare resources. Table 1 summarizes recommendations for authors and journal editors and reviewers that may improve the quality of methodology and reporting of trials. Journals and reviewers should require all clinical investigators submitting RCTs to report whether an a priori power analysis was performed.

\section{Compliance with Ethical Standards}

Conflict of interest The authors declare that they have no conflicts of interest to disclose.

Human Participants and Animals Rights This article does not contain any studies with human participants or animals performed by any of the authors.

Informed Consent For this type of study, informed consent is not required.

\section{References}

1. Portney LG, Watkins MP (2008) Foundations of clinical research: applications to practice, 3rd edn. Pearson/Prentice Hall, Upper Saddle River, NJ

2. Abdullah L, Davis DE, Fabricant PD, Baldwin K, Namdari S (2015) Is there truly "no significant difference"? Underpowered randomized controlled trials in the orthopaedic literature. J Bone Joint Surg Am. 97(24):2068-2073. https://doi.org/10.2106/JBJS. O.00012 (PMID: 26677241)

3. Agha RA, Camm CF, Doganay E, Edison E, Siddiqui MR, Orgill DP (2014) Randomised controlled trials in plastic surgery: a systematic review of reporting quality. Eur J Plast Surg 37(2):55-62

4. Voineskos SH, Coroneos CJ, Ziolkowski NI, Kaur MN, Banfield L, Meade MO, Thoma A, Chung KC, Bhandari M (2016) A systematic review of surgical randomized controlled trials: Part I. Risk of bias and outcomes: Common pitfalls plastic surgeons can overcome. Plast reconstr surger. 137(2):696-706

5. Voineskos SH, Coroneos CJ, Ziolkowski NI, Kaur MN, Banfield L, Meade MO, Chung KC, Thoma A, Bhandari MA (2016) Systematic review of surgical randomized controlled trials: part 2 funding source, conflict of interest and sample size in plastic surgery. Plast Reconstr Surg. 137(2):453e-461e

6. Johnson ES, Brookhart MA, Myers JA. (2013) Study Size Planning. In: Velentgas P, Dreyer NA, Nourjah P, et al. (eds). Developing a Protocol for Observational Comparative Effectiveness Research: A User's Guide. Rockville (MD): Agency for Healthcare Research and Quality (US); Jan. Chapter 9.

7. Chung KC, Kalliainen LK, Spilson SV, Walters MR, Kim HM (2002) The prevalence of negative studies with inadequate statistical power: an analysis of the plastic surgery literature. Plast Reconstr Surg. 109(1):1-6 (discussion 7-8)

8. Ayeni O, Dickson L, Ignacy TA, Thoma A (2012) A systematic review of power and sample size reporting in randomized 
controlled trials within plastic surgery. Plast Reconstr Surg. 130(1):78e-86e

9. Samargandi OA (2020) Improving reporting of research articles in aesthetic plastic surgery. Aesthetic Plast Surg. 44(5):1935-1936

10. Boutron I, Altman DG, Moher D, Schulz KF, Ravaud P (2017) CON-SORT NPT Group CONSORT statement for randomized trials of nonpharmacologic treatments: a 2017 update and a consort extension for nonpharmacologic trial abstracts. Ann Intern Med. 167(1):40-47
11. von Elm E, Altman DG, Egger M, Pocock SJ, Gøtzsche PC, Vandenbroucke JP, Initiative STROBE (2008) The strengthening the reporting of observational studies in epidemiology (STROBE) statement: guidelines for reporting observational studies. J Clin Epidemiol 61(4):344-349

Publisher's Note Springer Nature remains neutral with regard to jurisdictional claims in published maps and institutional affiliations. 